\title{
Relações entre Atitude, Conceito E Utilidade da Estatística ${ }^{1}$
}

Claudette Maria Medeiros Vendramini ${ }^{2}$

Márcia Regina Ferreira de Brito ${ }^{3}$

\section{Resumo}

Pesquisas têm indicado atitudes negativas dos estudantes em relação à Estatística e dificuldades na aprendizagem dessa disciplina. Assim, avaliou-se a relação entre essas atitudes, o conceito e a utilidade da Estatística para 319 universitários que responderam a um questionário informativo e a uma escala de atitudes em relação à Estatística. Essa escala apresentou uma alta consistência interna. Poucos conseguiram definir Estatística e não apresentaram diferença de atitudes em relação àqueles que não conseguiram defini-la. A maioria considerou-a uma ferramenta útil, revelando ter atitudes mais positivas do que aqueles que não a consideraram útil. Esses resultados sugerem que sejam adotadas estratégias de ensino que revelem a utilidade da Estatística para que os estudantes desenvolvam atitudes mais positivas, melhorando o ensino-aprendizagem dessa disciplina.

Palavras-chave: Atitudes em relação à Estatística; Ensino de Estatística; Ensino Superior.

\section{Relationships Among the AtTitude, ConcePT and Usefulness of the Statistics}

\begin{abstract}
Researches have indicated students' negative attitudes towards Statistics and difficulties to learn it. Thus, this paper analyzes the relationships between the attitudes towards Statistics, its concept and its usefulness for 319 university students who answered an informative questionnaire and a scale of attitudes towards Statistics. This scale shows a high internal consistence. The few subjects who were able to define Statistics did not present any differences in attitudes as compared to those who were not able to define it. The majority of the students who considered Statistics a useful tool revealed more positive attitudes than those ones who did not consider it useful. These results suggest that teaching strategies that reveal the usefulness of Statistics should be adopted to allow students to develop more positive attitudes, and, thus, improve its teaching-learning process.
\end{abstract}

Key words: Attitude towards Statistics; Teaching-learning Statistics; Higher education.

\section{INTRODUÇÃ̃o}

$\mathrm{O}$ ensino-aprendizagem de probabilidade e estatística cada vez mais ocupa um lugar importante nas Instituições de Ensino Superior devido à necessidade atual de profissionais capacitados em lidar com grande quantidade de informações, processadas em tempo mínimo, e com domínio de técnicas de análise de dados que fundamentem a tomada de decisões baseada na inferência de dados amostrais.

A educação estatística, principalmente para usuários de outras áreas de conhecimento, enfrenta sérios problemas por lidar com conceitos abstratos, usar notações e terminologias complexas e muitas vezes am- bíguas e confusas, ter a matemática como linguagem e lidar com problemas do mundo real que envolvem tomadas de decisões em condições de incerteza (Watts, 1991), implicando em inseguranças, medos, ansiedade, atitudes negativas em relação a esta disciplina.

Muitos estudantes ficam apreensivos quando enfrentam a expectativa de ter que cursar uma disciplina obrigatória de Estatística e freqüentemente já entram no curso com atitudes negativas em relação a essa disciplina ou desenvolvem estas atitudes durante as aulas (McCall, Mahidi e Belli, 1991; Wise, 1985).

Em pesquisas realizadas por vários professores,

${ }^{1}$ Parte da Tese de Doutorado da primeira autora, defendida na Faculdade de Educação da UNCAMP, sob a orientação da segunda autora.

${ }^{2}$ Bacharel em Estatística, Doutora em Educação pela FE-UNICAMP e docente da Universidade São Francisco. E-mail: cvendramini@uol.com.br

${ }^{3}$ Bacharel em Psicologia, Livre-Docente pela UNICAMP.E-mail: $\underline{\text { mbrito@unicamp.br }}$ 
Brito (1996) constatou que as afirmações dos alunos a respeito dos sentimentos negativos gerados pelas disciplinas "matemáticas" eram constantes, e que algumas dessas disciplinas eram difíceis e aversivas. Segundo a autora, este fato parece mostrar que as pessoas, de um modo geral, e os alunos de segundo grau, em particular, não gostam da Matemática e das atividades que envolvem a Matemática, sentimento que, aparentemente, se cristalizaria na universidade.

Em experiência relatada por Vendramini (2000) com alunos ingressantes no curso de Psicologia, foi possível constatar que esses alunos tinham uma barreira inicial quanto à disciplina Estatística, ou mesmo atitudes negativas em relação a ela. Com o trabalho desenvolvido durante o período letivo, os alunos passavam a compreender melhor o significado e a importância da Estatística para o desempenho de suas futuras profissões, mas continuavam apresentando dificuldades para atingir um bom desempenho acadêmico na disciplina.

Esse desempenho acadêmico, em Estatística, pode estar relacionado a experiências anteriores de aprendizagem. Nesse sentido, professores universitários freqüentemente têm declarado que seus alunos não estão preparados para enfrentar as exigências do curso superior, e possivelmente isto pode ser atribuído ao mau aproveitamento escolar nos cursos de ensino fundamental e médio. $\mathrm{O}$ bom desempenho acadêmico dos alunos pressupõe o domínio de certas habilidades, como estabelecer relações entre variáveis, distinguir observações de inferências, raciocinar hipoteticamente, capacidade de argumentação, dentre outras. Quando insuficientes, impedem-nos de pensar criticamente e inviabilizam, principalmente, a construção de novos conhecimentos (Pugh e Pawan, 1991).

Segundo Gal e Ginsburg (1994), as atitudes negativas em relação à Estatística são um cenário familiar a outros pesquisadores como Perney e Ravid (1991), segundo os quais a Estatística é vista como um obstáculo para a obtenção de diplomas de muitos universitários, que retardam o máximo possível a matrícula nesta disciplina. Segundo esses autores, os professores universitários de disciplinas referentes à pesquisa e estatística já estão familiarizados com o alto nível de ansiedade apresentado pelos estudantes.

No entanto, vários pesquisadores mostraram que as atitudes de universitários podem ser modificadas mediante algumas estratégias utilizadas para o ensino da
Estatística. Garfield (1994b) explorou esse aspecto em uma pesquisa realizada sobre as atitudes de 917 alunos de professores treinados nos cursos intensivos de um projeto denominado Quantitative Literacy Project $(Q L P)$. Os resultados mostraram que esses alunos tiveram atitudes mais positivas em relação à aprendizagem de Estatística. Um aumento dessas atitudes positivas em vários níveis sugeriu que os tópicos podem ser aceitos mais facilmente e podem, portanto, ser utilizados mais apropriadamente no ensino superior.

Entre as pesquisas que discutiram a importância da Estatística, suas aplicações e as habilidades necessárias para compreendê-la, Huberty, Dresden e Bak (1993) sugeriram que os estudantes sejam testados em três domínios da disciplina: os cálculos, o conhecimento proposicional e a compreensão conceitual. Os resultados de seus estudos mostraram que os estudantes têm uma fraca compreensão conceitual de Estatística e que, por conseguinte, é necessário que os professores os encorajem a realizar conexões entre idéias e habilidades que os levem a desenvolver maior habilidade para utilizar métodos estatísticos no futuro.

Com o fácil acesso a pacotes estatísticos computacionais, torna-se possível que usuários leigos usem técnicas estatísticas indiscriminadamente, sem o domínio dos conceitos básicos e sem uma análise cuidadosa da adequação dessas técnicas aos dados disponíveis, ou, ainda, que consumam informações estatísticas sem uma análise crítica das mesmas (Dallal, 1990, conforme citado por Cazorla, Silva, Vendramini e Brito, 1999).

Considerando a compreensão conceitual um domínio necessário e fundamental para a utilização adequada de técnicas estatísticas e a atitude positiva um elemento importante para facilitar a aprendizagem de conceitos estatísticos, o presente trabalho objetivou avaliar as atitudes em relação à Estatística e suas relações com a aprendizagem de conceitos estatísticos e, assim, poder entender melhor esse processo de ensino-aprendizagem.

Com base nas várias definições de atitudes, apresentadas por diferentes autores (Stagner, 1937; Guilford, 1954; Shrigley, Koballa e Simpson, 1988; Brito, 1996) considerou-se atitude como sendo: uma disposição mental, dirigida a objetos, eventos ou pessoas, que assume diferente direção e intensidade de acordo com as experiências do indivíduo, e que apresenta componentes do domínio afetivo, cognitivo e motor. 
Essa autora considera a atitude em relação à Matemática adaptada à definição de Stagner (1937), que caracterizaria essa atitude em particular por um objeto (a Matemática), uma direção (positiva ou negativa) e uma intensidade (gostar da ou ter aversão à Matemática).

Nesse trabalho, portanto, a atitude foi caracterizada, assim como pelos autores citados, por um objeto (a Estatística), uma direção (positiva ou negativa) e uma intensidade (gostar da ou ter aversão à Estatística).

Os conceitos básicos considerados referiram-se ao conceito e utilidade da Estatística, por serem essenciais para a aprendizagem de outros conceitos relacionados à Estatística.

Assim, espera-se que os resultados desse trabalho possam orientar professores de Estatística, ou de outras áreas, na busca de novas metodologias de ensino que facilitem a aprendizagem dessa disciplina, muito solicitada pela sociedade atual.

\section{MÉTODO}

\section{Sujeitos}

De um total de 29 cursos de uma universidade particular do interior do Estado de São Paulo, foram selecionados, intencionalmente, dois da área de Ciências Humanas (Psicologia, Administração), dois da área de Ciências Exatas (Ciência da Computação e Engenharia Mecânica-Automação e Sistemas Mecatrônica) e dois da área de Ciências da Saúde (Farmácia e Medicina), totalizando 319 sujeitos, sendo $43,3 \%$ do gênero masculino e $56,7 \%$ do feminino, com idades variando de 18 a 35 anos, média igual a 21,7 anos e desvio padrão igual a 3,7 anos. Os sujeitos eram alunos ingressantes na universidade no período de 1996 a 1998, regularmente matriculados, cursando a disciplina Estatística em 1998.

\section{Instrumentos}

Os dados foram obtidos a partir de dois instrumentos: um Questionário do aluno (Anexo 1) composto por 20 questões (ano de ingresso, série, curso, idade, gênero faculdade, período, escolaridade e profissão dos pais, reprovações em Estatística, conceito e utilidade da Estatística, disciplina já reprovada, disciplina de que menos gosta e de que mais gosta) e uma Escala de Atitudes em relação à Estatística (EAE) composta por 20 proposições do tipo Likert (Anexo 2), elaborada por Aiken (1970) para medir atitudes em relação à Matemática, traduzida, testada e validada por Brito em 1996, alterada e validada por Cazorla, Silva, Vendramini e Brito (1999), mudando a palavra Matemática para Estatística, acrescida de uma proposição complementar.

As dez proposições positivas e as dez negativas, referiram-se à Estatística "em si" e não a situações de ensino-aprendizagem ou outros fatores. A proposição complementar de número 21 teve a finalidade de verificar a auto-percepção do universitário em relação ao próprio desempenho em Estatística e foi analisada separadamente.

A escala EAE contou com quatro alternativas: Concordo Totalmente, Concordo, Discordo, Discordo Totalmente, pontuadas com 4, 3, 2, 1 quando as proposições eram positivas e 1, 2, 3, 4 quando negativas. $\mathrm{O}$ máximo de pontos que podia ser obtido na escala de atitudes era de 80 e o mínimo de 20, indicando, respectivamente, atitudes mais positivas e mais negativas.

Para sua validação, a escala foi aplicada a 1.154 sujeitos de 15 cursos de graduação de duas universidades do estado de São Paulo, que cursavam a disciplina Estatística em 1998. Os resultados mostraram que a escala EAE tem confiabilidade e validade satisfatórias. O coeficiente alfa de Cronbach foi 0,95 sugerindo uma alta consistência interna da escala. A análise fatorial de componentes principais com rotação varimax indicou dois fatores ortogonais com autovalores maiores que um e explicando a $61,2 \%$ da variância total, sendo o primeiro fator explicando $51,5 \%$, mostrando a sua dominância.

\section{Procedimento}

Os instrumentos foram aplicados, por professores colaboradores que não estavam lecionando Estatística em 1998 para as turmas selecionadas. As aplicações foram feitas em novembro de 1998, durante o horário de aula, na presença do professor colaborador. Cada instrumento foi precedido de um texto que expôs os objetivos e motivos principais da pesquisa.

\section{ResultadoS E DISCUSS̃̃0}

Os dados foram analisados a partir da utilização do programa STATISTICA (1997), versão 5.1 da StatSoft, adotando o nível de significância de 0,05 para 
a tomada de decisões baseada nas análises estatísticas realizadas.

Para a categorização dos sujeitos quanto ao conceito de Estatística os dados foram agrupados segundo alguns dos atributos definidores de Estatística, a saber: coleta, organização e interpretação dos dados; representação dos dados de forma descritiva, tabular ou gráfica; cálculo de medidas que expressam de forma resumida as características de uma amostra; comparação de dados amostrais; cálculo de estimativas; probabilidade e inferência de dados amostrais para a população.

Considerando esses atributos, os dados foram agrupados nas seguintes categorias: (1) Identifica características do conceito de Estatística; (2) Identifica características relacionadas às operações matemáticas; (3) Descreve uma utilidade para a Estatística (4) Descreve um motivo para o estudo de Estatística; (5) Outras características ou comentários que não refletem o conceito de Estatística.

Para a categorização dos resultados foi respeitada a predominância das respostas dos sujeitos em cada categoria segundo o seguinte critério: categoria 1, quando o sujeito indicava pelo menos um dos atributos definidores do conceito de Estatística; categoria 2, quando o sujeito se referia às operações matemáticas, predominantemente; categoria 3, quando o sujeito citava pelo menos uma utilidade; categoria 4, quando o sujeito citava pelo menos um motivo; categoria 5 em outro caso.

Desta maneira foi possível verificar como os sujeitos referiram-se ao termo "Estatística".

Quanto às atitudes as variáveis observadas foram as vinte proposições da escala de atitudes em relação à Estatística. Considerou-se como válidas as escalas com no mínimo dezoito proposições respondidas, para que fosse possível o preenchimento das duas ou menos respostas em branco com a média das proposições respondidas pelo próprio sujeito nessa escala.

\section{Caracterização da amostra}

Os sujeitos diferiram significativamente entre as três áreas de conhecimento em relação ao gênero $\left(c^{2}(2)=86,208\right.$ e $\left.p<0,0001\right)$. A área de Exatas foi predominantemente do gênero masculino enquanto a de Humanas e da Saúde predominantemente do gênero feminino.
Quanto às idades a prova de Kruskal-Wallis não acusou diferença significativa entre os três grupos $(H(2,317)=0,8945 ; p=0,6394)$, embora a área de Humanas tenha apresentado maior idade média (22,1 anos) e maior variabilidade (desvio padrão $=4,4$ anos).

A área da Saúde apresentou maior porcentagem de pais $(31,8 \%)$ e de mães $(27,1 \%)$ com nível superior, por agregar alunos do curso de Medicina, que é o curso mais caro da universidade, além de ser o de maior concorrência no processo seletivo de ingresso no curso superior (vestibular). As diferenças entre as áreas foram estatisticamente significativas, para os pais $\left(c^{2}(4)=15,693 ; p=0,0035\right)$ e para as mães $\left(c^{2}(4)=10,723 ; p=0,0299\right)$.

Aproximadamente $50,0 \%$ dos sujeitos responderam ser filhos de pais e de mães que cursaram até o primeiro grau, $30,0 \%$ até o segundo grau e $20,0 \%$ o curso superior.

Quanto aos estudos, a maioria dos sujeitos não respondeu quanto à reprovação em Estatística $(95,9 \%)$. Entre os respondentes a área da Saúde foi a de maior porcentagem de sujeitos que haviam sido reprovados nesta disciplina $(11,0 \%)$.

Entre as disciplinas citadas pelos sujeitos como aquelas de que menos gostam, a Estatística foi citada em maior proporção na área de Humanas $(17,6 \%)$, seguida pela área de Exatas $(8,6 \%)$ e em menor proporção na área de Saúde (1,9\%), sendo significativas as diferenças entre três áreas de conhecimento $p<0,0001)$.

Entre as disciplinas citadas pelos sujeitos como aquelas preferidas, a Estatística foi citada em proporção inferior a 2,5\% em todas as áreas de conhecimento $(2,3 \%$ para Humanas, $0 \%$ para Exatas e $1,9 \%$ para Saúde). Também a Matemática foi citada em pequena proporção com valores inferiores a 11,0\% (10,7\% para Humanas, 8,6\% para Exatas e 0,9\% para Saúde).

\section{Conceito e utilidade da estatística}

Em geral, as pessoas referem-se ao termo Estatística apenas no sentido da organização e descrição dos dados, sem considerar o aspecto essencial que é o de proporcionar inferências que permitam conclusões que transcendam os dados obtidos inicialmente.

As questões 15 (Que idéia você tem da Estatística), 16 (Por que motivo estudar Estatística) e 17 
(Cite alguns exemplos de situações do cotidiano em que a Estatística pode ser aplicada), constantes do Questionário do Aluno (Anexo 1), permitiram identificar o nível de formação conceitual de Estatística e assim atingir o objetivo de verificar se aqueles que sabiam o significado e a importância da Estatística teriam atitudes mais positivas em relação a essa disciplina.

Os resultados revelaram que $24,5 \%$ dos sujeitos identificaram características do conceito de Estatística no nível descritivo ou no inferencial e $10,0 \%$ revelaram não ter identificado características desse conceito.

Ao responder à questão quinze, 29,2\% dos sujeitos escreveram sobre os motivos que os levaram a estudar Estatística sem fazer referência ao conceito.

Da mesma maneira, 16,3\% escreveram sobre a utilidade da Estatística. Nestes casos, não foi possível verificar se os sujeitos identificavam ou não características do conceito de Estatística.

Embora poucos tenham expressado não entender corretamente o significado da palavra Estatística, $80,3 \%$ declararam ter algum motivo, diferente da obrigatoriedade de cursar a disciplina, para estudá-la. Este fato pode ter refletido positivamente nas atitudes dos sujeitos com relação a esta disciplina.

A maioria desses sujeitos descreveu exemplos do cotidiano para a utilidade da Estatística, revelando que a maioria considerou a Estatística como uma ferramenta útil.

Com relação à concepção que possuem, 24,7\% vêem na Estatística uma ferramenta útil para sua carreira profissional, citando exemplos relacionados à atual área de estudo e ao futuro campo de trabalho, tais como: análise de produção, qualidade, vendas, estudo de processos industriais, para a Engenharia; estatísticas de lucro, prejuízo, gastos, balancetes bancários, para Administração; estatísticas populacionais ou de saúde, para Farmácia e Medicina e o exemplo testes psicológicos para a Psicologia.

As proporções de sujeitos que identificam e aquelas que não identificam características do conceito de Estatística diferem entre as áreas de conhecimento $\left(c^{2}(2)=17,787 ; p=0,0001\right)$, sendo a área de Humanas aquela que apresentou maior porcentagem de sujeitos que descreve alguma característica do conceito de Estatística. Diferem, também, quanto ao motivo expresso para estudar Estatística $\left(c^{2}(2)=7\right.$, 409; $p=0,0246$ ), sendo a área de Exatas aquela que apresentou maior porcentagem de sujeitos com motivo para estudar Estatística. Já a proporção de sujeitos que expressam utilidades para a Estatística não difere entre as áreas de conhecimento $\left(c^{2}(2)=1,170\right.$; $p=0,5572$ ).

Quanto às diferenças entre os gêneros concluiu-se que as proporções de sujeitos que expressam e que não expressam atributos do conceito de Estatística são estatisticamente significativas $\left(c^{2}(1)=8,997 ; p=0,0027\right)$, sendo o gênero masculino aquele que apresentou a maior porcentagem de sujeitos que expressa tais atributos. A utilidade expressa pelos sujeitos do gênero masculino também foi significativamente superior ao do feminino $\left(c^{2}(1)=5,413 ; p=0,0200\right)$. Não houve diferença significativa entre os gêneros quanto ao motivo expresso pelos sujeitos para estudar Estatística $\left(c^{2}(1)=0,136 ; p=0,7123\right)$.

É importante lembrar que os sujeitos que participaram do presente estudo responderam aos instrumentos no final do ano letivo em que estavam cursando a disciplina Estatística. Esta podia ser cursada na primeira, segunda ou terceira série, dependendo do curso. Como revelado no teste Qui-quadrado, quando os sujeitos foram agrupados de acordo com a série não houve diferença de proporções de sujeitos que expressaram motivos $\left(c^{2}(2)=0,321\right.$; $p=0,8517)$ e utilidade $\left(c^{2}(2)=3,830 ; p=0,1474\right)$ para a Estatística. Mas houve diferença significativa entre as séries quando a variável formação conceitual de Estatística foi considerada $\left(c^{2}(2)=9,865 ; p=0,0072\right)$, sendo a primeira série aquela com maior portagem de sujeitos que expressou atributos do conceitos de Estatística.

Os coeficientes de contingência calculados revelaram que não existia relação entre as variáveis "conceito" e "utilidade" da Estatística ( $C=0,065$; $p=0,2443$ ), e que a maioria dos sujeitos encontraram no mínimo uma utilidade para a Estatística, tanto no grupo dos sujeitos que souberam definir o que é "Estatística" $(92,3 \%)$ quanto no grupo dos que não souberam defini-la $(87,5 \%)$. Pelo resultado da prova não paramétrica de Fisher foi possível afirmar que não existiam diferenças significativas entre aqueles que acreditavam na utilidade da Estatística e os sujeitos que conseguiram definir "o que é Estatística" e aqueles que não conseguiram ( $p=0,3062)$. 


\section{ATITUDE E SUA RELAÇÃO COM 0 CONCEITO E A UTILIDADE DA ESTATÍSTICA}

A soma total de pontos na escala de atitudes, adotada nesse trabalho, podia variar de 20 (atitudes mais negativas) a 80 (atitudes mais positivas). Outros autores aplicaram escalas de atitudes semelhantes à essa, com diferentes números de itens, como os autores Roberts e Bilderback (1980), Roberts e Reese (1987), Glencross e Cherian (1992, 1995).

Os escores dos sujeitos na escala de atitudes em relação à Estatística apresentaram média de 52,2 pontos e mediana igual a 52 e um desvio padrão de 8,7 pontos, representando a variabilidade destes escores em torno dessa média, revelando que as atitudes dos sujeitos foram mais positivas que negativas.

As atitudes dos sujeitos dessa pesquisa são mais positivas do que o grupo de 1.154 estudantes da pesquisa de Cazorla et al. (1999) e com menor variabilidade de resultados (média $=50,5$ e desvio padrão=10,2).

A escala de atitudes apresentou uma alta consistência interna geral, alfa de Cronbach igual a 0,9282, variando de 0,8956 a 0,9514 nos diversos grupos estudados (área de conhecimento, gênero, série, faixa etária, escolaridade dos pais e das mães, autopercepção de desempenho em Estatística).

Pela análise de variância (ANOVA) foi possível verificar a existência de diferenças significativas entre as médias das variáveis pesquisadas, nos vários grupos de estudo.

As atitudes não diferiram significativamente quando os sujeitos foram agrupados de acordo com a área de conhecimento $(F(2,316)=2,718 ; p=0,0675)$. Nas três áreas de conhecimento os sujeitos apresentaram atitudes mais positivas que negativas em relação à Estatística.

Tabela 1 - Estatísticas descritivas e confiabilidade da escala de atitudes em relação à Estatística de acordo com os grupos de variáveis

\begin{tabular}{|c|c|c|c|c|c|}
\hline Variável & Grupo & $\begin{array}{l}\text { Casos } \\
\text { válidos }\end{array}$ & Média & $\begin{array}{l}\text { Desvio } \\
\text { Padrão }\end{array}$ & $\begin{array}{l}\text { Alfa de } \\
\text { Cronbach }\end{array}$ \\
\hline \multirow[t]{3}{*}{ Área de conhecimento } & Humanas & 131 & 51,53 & 9,15 & 0,9335 \\
\hline & Exatas & 81 & 51,15 & 7,34 & 0,8978 \\
\hline & Saúde & 107 & 53,75 & 8,87 & 0,9351 \\
\hline \multirow[t]{2}{*}{ Gênero } & Masculino & 138 & 52,17 & 8,95 & 0,9157 \\
\hline & Feminino & 181 & 52,18 & 9,22 & 0,9358 \\
\hline \multirow[t]{3}{*}{$\overline{\text { Série }}$} & Primeira & 194 & 51,21 & 8,32 & 0,9184 \\
\hline & Segunda & 87 & 55,06 & 9,22 & 0,9412 \\
\hline & Terceira & 38 & 50,50 & 7,82 & 0,9181 \\
\hline \multirow[t]{5}{*}{ Idade (anos) } & Até 20 & 143 & 51,92 & 7,62 & 0,9020 \\
\hline & De 21 a 25 & 132 & 52,08 & 9,44 & 0,9416 \\
\hline & De 26 a 29 & 22 & 54,05 & 9,50 & 0,9389 \\
\hline & Mais de 29 & 19 & 52,74 & 9,69 & 0,9514 \\
\hline & Em branco & 3 & - & - & - \\
\hline \multirow[t]{4}{*}{ Escolaridade do pai } & Até $1^{\circ}$ grau & 150 & 52,11 & 8,55 & 0,9233 \\
\hline & $2^{\circ}$ grau & 102 & 51,46 & 8,68 & 0,9276 \\
\hline & Superior & 59 & 53,66 & 8,92 & 0,9393 \\
\hline & Em branco & 8 & - & - & - \\
\hline \multirow[t]{4}{*}{ Escolaridade da mãe } & Até $1^{\circ}$ grau & 166 & 51,95 & 8,64 & 0,9299 \\
\hline & $2^{\circ}$ grau & 93 & 50,02 & 7,94 & 0,9059 \\
\hline & Superior & 57 & 56,44 & 8,69 & 0,9374 \\
\hline & Em branco & 3 & - & - & - \\
\hline \multirow{2}{*}{$\begin{array}{l}\text { Autopercepção do } \\
\text { desempenho em Estatística }\end{array}$} & Ruim & 122 & 48,55 & 7,67 & 0,8956 \\
\hline & Bom & 197 & 54,42 & 8,52 & 0,9337 \\
\hline Total geral & & 319 & 52,18 & 8,68 & 0,9282 \\
\hline
\end{tabular}


Quando agrupados de acordo com a série, as atitudes dos sujeitos diferiram significativamente $(F(2,316)=6,954 ; p=0,0011)$. Nas três séries, as atitudes em relação à Estatística foram mais positivas que negativas. O teste de Tukey (DHS) mostrou que as atitudes dos sujeitos da primeira série foram significativamente menos positivas que aquelas apresentadas pelos da segunda série, e estas significativamente mais positivas que as apresentadas pelos sujeitos da terceira série $(p=0,0063)$. Não houve diferença entre as atitudes da primeira e da terceira séries $(p=0,6382)$. positivas que negativas quando comparados aos sujeitos do gênero feminino.

A análise de variância apontou que os sujeitos dos vários grupos de idade não apresentaram diferenças de atitudes estatisticamente significativas $(F(3$, 312 ) $=0,413, p=0,7440)$, embora possa ser verificada uma tendência às atitudes mais positivas no grupo de sujeitos com idades mais elevadas.

$\mathrm{O}$ agrupamento dos sujeitos de acordo com a escolaridade dos pais e das mães permitiu concluir que as atitudes dos sujeitos não diferiram significativamente nos di-

Tabela 2 - Análise de variância das atitudes em relação à Estatística de acordo com os grupos de variáveis

\begin{tabular}{|c|c|c|c|c|c|}
\hline \multirow[b]{2}{*}{ Variável } & \multicolumn{2}{|c|}{$\begin{array}{c}\text { Homogeneidade } \\
\text { de variância } \\
\end{array}$} & \multicolumn{2}{|l|}{ ANOVA } & \multirow{2}{*}{$\begin{array}{l}\text { Grupos com } \\
\text { diferenças } \\
\text { significativas }\end{array}$} \\
\hline & $\mathrm{F}^{1}$ & $p$ & $\mathrm{~F}\left(\mathrm{gl}_{\text {entre }}, \mathrm{gl}_{\text {dentro }}\right)$ & $p$ & \\
\hline Áreas de conhecimento & 2,615 & 0,0747 & $F(2,316)=2,718$ & 0,0675 & - \\
\hline Série & 1,273 & 0,2815 & $F(2,316)=6,954$ & 0,0011 & $\begin{array}{l}1^{\mathrm{a}} \text { e } 2^{\mathrm{a}} \text { séries } \\
2^{\mathrm{a}} \text { e } 3^{\mathrm{a}} \text { séries }^{*}\end{array}$ \\
\hline Gênero & 1,829 & 0,1772 & $F(2,317)=0,000$ & 0,9977 & - \\
\hline Faixa etária & 2,097 & 0,1006 & $F(2,312)=0,413$ & 0,7440 & - \\
\hline Escolaridade dos pais & 0,624 & 0,5362 & $F(2,308)=1,220$ & 0,2968 & - \\
\hline Escolaridade das mães & 0,550 & 0,5773 & $F(2,313)=10,340$ & 0,0000 & $\begin{array}{l}1^{\circ} \text { grau e Superior } \\
2^{\circ} \text { grau e Superior }\end{array}$ \\
\hline Matéria de que menos gosta & 3,687 & 0,0267 & $F(2,208)=27,093$ & 0,0000 & $\begin{array}{l}\text { Matemática e outras }{ }^{* * *} \\
\text { Estatística e outras }{ }^{* * *}\end{array}$ \\
\hline Matéria de que mais gosta & 2,469 & 0,0869 & $F(2,231)=8,110$ & 0,0004 & $\begin{array}{l}\text { Matemática e outras * } \\
\text { Estatística e outras * }\end{array}$ \\
\hline $\begin{array}{l}\text { Auto-percepção do } \\
\text { desempenho em Estatística }\end{array}$ & 0,543 & 0,4619 & $F(1,317)=38,570$ & 0,0000 & Ruim e Bom ${ }^{\star * *}$ \\
\hline
\end{tabular}

${ }^{1} \mathrm{~F}$ do teste de Levene para homogeneidade de variâncias

"Diferenças significativas $(p<0,05) ;{ }^{*}$ Diferenças muito significativas $(p<0,01)$

${ }^{* *}$ Diferenças altamente significativas $(p<0,001)$

O agrupamento dos sujeitos de acordo com o gênero revelou que não houve diferença significativa entre as atitudes dos sujeitos do gênero masculino e do feminino $(F(1,317)=0,000 ; p=0,9977)$. Esses resultados são semelhantes aos encontrados por Waters, Martelli, Zakrajset e Popovic (1989), Cazorla et al. (1999), embora outras pesquisas apontem diferenças de atitudes quanto ao gênero, tanto em relação à Estatística (Roberts e Saxe, 1982; Silva et al.,1999) quanto em relação à Matemática (Brito, 1996) e indiquem que os sujeitos do gênero masculino têm atitudes mais ferentes grupos de escolaridade dos pais $(F(2,308)=1,220$; $p=0,2968)$. As atitudes em relação à Estatística foram mais positivas que negativas nesses grupos, mas diferiram significativamente entre os grupos de escolaridade das mães, $(F(2,313)=10,340 ; p<0,0001)$. O teste de Tukey (DHS) revelou que as atitudes dos sujeitos com mães que possuem o nível superior foram mais positivas que o grupo de sujeitos com mães que cursaram até o segundo grau, e mais positivas que o do grupo de sujeitos com mães que cursaram até o primeiro grau, conforme dados apresentados na Tabela 2. 
As diferenças entre as médias das atitudes em relação à Estatística de acordo com a preferência de disciplinas foram significativas, tanto entre os grupos disciplinas de que menos gostavam $(F(2,208)=27,093$; $p<0,0001)$ quanto entre os grupos de disciplinas de que mais gostavam $(F(2,231)=8,110 ; p=0,0004)$.

Os resultados apontaram que a média das atitudes dos sujeitos que menos gostavam de Matemática foi inferior à apresentada pelo grupo que afirmou gostar menos de outras disciplinas. Esse resultado foi verificado também para a média das atitudes dos sujeitos que menos gostavam de Estatística.

Quanto aos sujeitos que declararam que a matéria de que mais gostavam era a Estatística, estes tiveram atitudes significativamente superiores às dos outros grupos $(F(2,231)=8,110 ; p=0,0004)$. Foram encontradas diferenças significativas também entre a média de atitudes dos sujeitos que declararam preferir a Estatística e a dos sujeitos que declararam preferir outras disciplinas. As atitudes destes sujeitos foram bem mais positivas que as encontradas nos outros dois grupos.

Para avaliar a relação entre as atitudes e a autopercepção do desempenho em Estatística, foi formulada a seguinte proposição: Eu não tenho um bom desempenho em Estatística, sendo que o aluno tinha quatro alternativas de resposta: concordo totalmente, que seria indicador de uma autopercepção de desempenho muito ruim; concordo, indicador de ruim; discordo, de bom e discordo totalmente, de muito bom. Estas alternativas foram agrupadas em duas categorias: sujeitos com uma autopercepção de bom desempenho em Estatística e sujeitos com autopercepção de desempenho ruim.

A análise de variância dos valores da escala de atitudes pela autopercepção foi significativa $(F(1,317)=38,570$; $p<0,0001$, demonstrando que a média das atitudes dos sujeitos que perceberam ter um bom desempenho em Estatística foi superior à dos que perceberam ter um desempenho ruim.

Os resultados das variáveis quantitativas foram agrupados em categorias para que fosse possível compará-las com as variáveis categóricas conceito e utilidade da Estatística. Assim, foram considerados os sujeitos com atitudes positivas, aqueles que obtiveram um total de pontos na escala de atitudes maior ou igual a 52, e os sujeitos com atitudes negativas, aqueles que obtiveram pontuação inferior a 52.

A análise mostrou que não existia relação entre o "conceito" e a "atitude" em relação à Estatística
$(C=0,074 ; p=0,1886)$. Não foram observadas diferenças significativas de atitudes entre os sujeitos que conseguiram definir "o que é Estatística" e aqueles que não conseguiram defini-la $(p=0,1950)$. No entanto, os resultados mostraram uma correlação positiva entre a "utilidade" e a "atitude" em relação à Estatística ( $C=0,159 ; p=0,0041$ ), sendo que $95 \%$ dos sujeitos com atitudes positivas citaram pelo menos uma utilidade para a Estatística, enquanto no grupo de sujeitos com atitudes negativas essa porcentagem $(83,8 \%)$ foi significativamente inferior $(p=0,0044)$.

\section{CONCLUSÕES E CONSIDERAÇÕES FINAIS}

O presente estudo buscou verificar as relações entre as atitudes em relação à Estatística e o conceito e a utilidade da Estatística de universitários de diferentes áreas de conhecimento, gênero, idade e escolaridade dos pais.

Os resultados revelaram que poucos sujeitos $(24,5 \%)$ demonstraram conhecer o conceito de "Estatística", a maioria não conseguiu identificar características do conceito; no entanto, $80,3 \%$ declararam ter motivo para estudá-la e 90,0\% consideraram a Estatística uma ferramenta útil.

Quando foram verificadas as relações entre as variáveis estudadas, não se encontraram diferenças significativas das atitudes em relação à Estatística entre os sujeitos que conseguiram identificar características do conceito de Estatística e aqueles que não conseguiram identificar nenhuma característica de sua definição. No entanto, a porcentagem de sujeitos com atitudes positivas, que citaram pelo menos uma utilidade para a Estatística, foi significativamente superior à porcentagem de sujeitos com atitudes negativas.

Outros pesquisadores encontraram resultados semelhantes (Silva, Cazorla e Brito, 1999), em estudos realizados com alunos de graduação de várias áreas de conhecimento, e constataram que os estudantes que consideravam a Estatística uma ferramenta importante e confiável, também apresentavam atitudes positivas. Em uma outra pesquisa, Feijoo (1991) verificou que os sujeitos da área de Humanas que reconheciam a importância e a utilidade da Estatística e que apontavam a utilidade da Estatística, também tendiam a ter atitudes mais positivas em relação a esta disciplina.

Na presente pesquisa, a média dos escores dos sujeitos na escala de atitudes em relação à Estatística foi 
de 52,2 pontos, sendo considerados sujeitos com atitudes positivas aqueles que obtiveram pontuação acima dessa média, e sujeitos com atitudes negativas aqueles que obtiveram pontuação abaixo dessa média. Os resultados revelaram que os sujeitos apresentaram atitudes positivas, na maioria dos grupos estudados, sendo que as atitudes mais positivas foram observadas: (1) no grupo de sujeitos cujas mães estudaram até o nível superior (média=56,44), (2) no grupo de sujeitos que estavam cursando a segunda série (média $=55,06)$ e (3) no daqueles com autopercepção de um bom desempenho em Estatística (média=54,42).

Vale ressaltar que as atitudes em relação à Estatística estão relacionadas às atitudes em relação à Matemática, como revelaram as pesquisadoras Silva, Cazorla, Vendramini e Brito (2000) em uma pesquisa realizada com trezentos e trinta universitários. Assim, a média das atitudes dos sujeitos que declararam gostar menos de Matemática e, também, daqueles que declararam gostar menos de Estatística, foram significativamente inferiores à apresentada pelo grupo que afirmou gostar menos de outras disciplinas, revelando uma possível associação entre essas atitudes.

Há alguns anos, o ensino da Estatística vem sendo objeto de estudo de vários pesquisadores (Cazorla et al., 1999; Gal e Ginsburg, 1994; McCall, Madjidi e Belli e, 1991; Rosenthal, 1992; Wise, 1985) preocupados com a percepção dos alunos em relação à essa disciplina e à ansiedade desses alunos ao enfrentar a expectativa de ter que cursar a disciplina Estatística, obrigatória na maioria dos cursos de graduação. Geralmente os alunos iniciam as aulas com atitudes negativas em relação a essa disciplina ou as desenvolvem no decorrer do período letivo.

Esses problemas de ordem afetiva na aprendizagem da Estatística, se negativos, podem dificultar a aprendizagem dos conceitos dessa disciplina (Gal e Ginsburg, 1994) ou retardar o desenvolvimento do pensamento estatístico necessário para o desenvolvimento de muitas atividades profissionais.

Assim, resultados de pesquisas que estudam as relações entre o sentimento de gostar ou não da Estatística e a aprendizagem de seus conceitos podem contribuir para a melhoria do ensino da Estatística.

A análise da literatura mostra que vários autores (Gal e Ginsburg, 1994; Perney e Ravid, 1991; Peterson, 1991; Roberts e Reese, 1987; Roberts e Saxe, 1982; Wise, 1985; entre outros) obtiveram resultados que apontaram nessa direção e alguns, inclusive, apresen- taram propostas visando a melhoria das atitudes em relação à Estatística (Garfield, 1994a; Garfield, 1994b). Garfield (1994b) mostrou que os alunos de professores treinados em um projeto denominado Quantitative Literacy Project ( $Q L P$ ) obtiveram atitudes mais positivas em relação à aprendizagem de Estatística que alunos de professores não treinados. Sugeriu que os professores deveriam melhorar as atitudes dos alunos e que, como conseqüência, esses alunos aprenderiam mais facilmente os tópicos de Estatística.

Como as atitudes em relação à Estatística parecem ser decorrentes das atitudes em relação à Matemática (Silva et al., 2000) os professores de Estatística deveriam estar atentos à possibilidade de minimizar o "medo", a ansiedade que essa disciplina pode gerar. Assim, os professores de Estatística deveriam propor como um dos objetivos da disciplina a mudança de atitudes. Isso poderia ser feito por meio de técnicas de ensino que relacionassem a disciplina Estatística com os dados estatísticos utilizados no cotidiano e veiculados pelos jornais e a mídia em geral, constituindo-se numa "alavanca" geradora de motivação para a aprendizagem do conteúdo estatístico.

Uma outra fonte de consulta seriam os estudos desenvolvidos pelo grupo de pesquisa em Psicologia da Educação Matemática da Faculdade de Educação da UNICAMP, particularmente os referentes à Educação Estatística, tanto os concluídos (Cazorla et al., 1999; Cazorla et al., 2000; Silva, Cazorla e Brito, 1999; Silva et al., 2000; Vendramini et al., 2000) como os vários projetos em andamento.

Além desses, os trabalhos relacionados às atitudes e às habilidades matemáticas, desenvolvidos por esse grupo (Brito, 1996; Brito, Fini e Neumann García, 1994; Gonçalez, 1995; Brito, Gonçalez, e Vendramini, 1998; Gonçalez e Brito, 1996; Moron, 1998; Pirola, Silva e Vendramini, 1998; Spaletta, 1998; entre outros), poderiam estar subsidiando esta prática, tendo em vista a estreita relação existente entre a Estatística e a Matemática.

Concluindo, é importante alertar os professores de Estatística sobre a necessidade de elaborar programas visando o desenvolvimento de atitudes positivas em relação à Estatística e o desenvolvimento das habilidades matemáticas necessárias para a aprendizagem dessa disciplina, como, por exemplo, a leitura acurada e atenta da proposição do problema visando a obtenção da informação matemática (Vendramini, 2000).

A formação de profissionais competentes que saibam usar cuidadosa e adequadamente as técnicas es- 
tatísticas, com atitudes positivas em relação à essa disciplina, ao seu uso e à sua aplicação prática, deve nortear o ensino da Estatística em todas as áreas de conhecimento onde ela está inserida.

Os professores deveriam ser capazes de formular objetivos para a formação de atitudes, fornecer modelos exemplares na área, possibilitar que os alunos tenham experiências agradáveis em sala de aula, usar métodos de ensino criativos e variados e incentivar o aprimoramento individual do estudante, segundo a proposta de Klausmeier e Goodwin (1977).

O grande desafio é formar usuários de Estatística encorajados a utilizar as ferramentas estatísticas, capazes de reconhecer as ferramentas adequadas para solucionar seus problemas e que, mesmo não conseguindo operacionalizar sua solução, sejam capazes de buscar

\section{REFERÊNCIAS}

Aiken, L. R. (1970). Attitudes toward Mathematics. Review of Educational Research, 40 (4), 551-596.

Brito, M. R. F. (1996). Um estudo sobre as atitudes em relação à Matemática em estudantes de $1^{\circ}$ e $2^{\circ}$ graus. Tese de Livre Docência. Universidade Estadual de Campinas, Campinas, São Paulo.

Brito, M. R. F. (1998). Adaptação e validação de uma escala de atitudes em relação à Matemática. Zetetiké, 6(9), 109-162.

Brito, M. R. F., Fini, L. D. T. \& Neumann García, V. J. N. (1994). Um estudo exploratório sobre as relações entre o raciocínio verbal e o raciocínio matemático. ProProsições, 5 (1 [13]), 37-44.

Brito, M. R. F., Gonçalez, M. H. \& Vendramini, C. (1998). An exploratory study about verbal and mathematical abilities related to problem solving and attitudes. Abstracts of $24^{\text {th }}$ International Congress of Applied Psychology, San Francisco, Ca, USA, 116 - 117.

Cazorla, I. M., Silva, C. B., Vendramini, C. M. M. \& Brito, M. R. F. (1999). Adaptação e validação de uma escala de atitudes em relação à Estatística. Anais da Conferência Internacional: Experiências e perspectivas do auxílio com profissionais da área, que poderão orientálos na busca de soluções para esses problemas.

Uma sugestão para melhorar o ensino da Estatística é revelar ao estudante, logo no início das aulas, a aplicação das técnicas estatísticas na área de interesse, discutindo pesquisas já concluídas, e só então introduzir os conceitos teóricos necessários, visando atitudes mais positivas e o desenvolvimento de certas habilidades matemáticas. Os estudantes precisam ser desafiados e levados a elaborar uma pesquisa ligada ao seu campo de atuação atual, em que seja necessário utilizar as técnicas estatísticas de análise de dados. Pode-se contar, hoje, com a utilização de programas computacionais específicos como valioso auxílio para incentivar esses estudantes a pensar em termos probabilísticos.

ensino da Estatística, desafios para o século XXI. ABE, Florianópolis, Santa Catarina, versão preliminar, 4557.

Cazorla, I. M., Silva, C. B., Vendramini, C. M. M. \& Brito, M. R. F. (2000). Análise da relação entre os aspectos afetivos e cognitivos com o desempenho em Estatística. Anais do $14^{\circ}$ Simpósio Nacional de Probabilidade e Estatística. Caxambu, Minas Gerais, 162.

Dallal, G. E. (1990). Statistical computing package: dare we abandon their teaching to others? The American Statistician, 44(4), 265-266.

Feijoo, N. R. (1991). Estudio de las actitudes de los estudiantes universitarios hacia la Matematica y la Estadistica. Revista Intercontinental de Psicologia y Educación, 4(2), 69-83.

Gal, I. \& Ginsburg, L. (1994). The role of beliefs and attitudes in learning statistics: towards and assessment framework. Journal of Statistics Education [on line], 2(2). Available E-mail: archive@jse.ncsu.edu Message: send.

Garfield, J. (1994a). Beyond testing and grading: using assessment to improve student learning. Journal of 
Statistics Education [on line], 2(1). Available E-mail: archive@jse.ncsu.edu Message: send.

Garfield, J. (1994b). Student reactions to learning about probability and statistics: evaluating the quantitative literacy project. School Science and Mathematics, 94(2), 89-95.

Glencross, M. J. \& Cherian, V. I. (1992). Attitudes toward applied statistics of postgraduate students in education in Transkei. Psychological Reports, 70, 67-75.

Glencross, M. J. \& Cherian, V. I. (1995). Attitudes toward applied statistics of postgraduate students in education in the Lebowa region of South Africa. Psychological Reports, 77, 315-322.

Gonçalez, M. H. C. C. (1995). Atitudes (Des)Favoráveis com Relação à Matemática. Dissertação de Mestrado. Faculdade de Educação, Universidade Estadual de Campinas, Campinas, São Paulo.

Gonçalez, M. H. \& Brito, M. R. F. (1996). Atitudes (des) favoráveis em relação à matemática. Zetetiké, 4(6), 45-63.

Guilford, J. P. (1954). Psychometric Methods (2nd ed.). New York: McGraw Hill Book Company.

Huberty, C. J., Dresden, J. \& Bak, B. (1993). Relations among dimensions of statistical knowledge. Educational and Psychological Measurement, 53(2), 523-532.

Klausmeier, H. J. \& Goodwin, W. (1977). Manual de Psicologia Educacional - Aprendizagem e Capacidades Humanas. Tradução de Maria Célia Teixeira. São Paulo: Harper \& Row do Brasil.

McCall, C. H., Madjidi, F. \& Belli, G. (1991). The complexities of the teaching graduate students in educational administration introductory statistical concepts. PICTeachSt3, 2, 495-497.

Moron, C. F. (1998). Um estudo exploratório sobre as concepções e as atitudes dos professores de educação infantil em relação à Matemática. Dissertação de Mestrado. Faculdade de Educação, Universidade Estadual de Campinas, Campinas, São Paulo.
Perney, J. \& Ravid, R. (1991). The relationship between attitudes towards Statistics, math self-concept, test anxiety and graduate students' achievement in an introductory statistics course. Manuscrito não publicado, National College of Education, Evanston, IL. In Gal, I., \& Ginsburg, L. The role of beliefs and attitudes in learning statistics: towards and assessment framework. Journal of Statistics Education [on line], 2(2). Available E-mail: archive@ jse.ncsu.edu Message: send.

Peterson, I. (1991). Pick a sample. Science News, 140, 5658.

Pirola, A. N., Silva, M. V. \& Vendramini, C. M. M. (1998). Um estudo sobre a resolução de problemas em alunos universitários. III Congreso Iberoamericano de Educación Matemática, Caracas, Venezuela.

Pugh, S. L. \& Pawan, F. (1991). Reading, writing, and academic literacy. In: R. F. Flippo \& D. C. Caverly (Eds.). College Reading and Strategy Programs. Newark: IRA.

Roberts, D. M. \& Bilderback, E. W. (1980). Reliability and validity of a statistics attitudes survey. Educational and Psychological Measurement, 40, 235-238.

Roberts, D. M. \& Reese, C. M. (1987). A comparison of two scales measurement attitudes towards statistics. Educational and Psychological Measurement, 47, 759-764.

Roberts, D. M. \& Saxe, J. E. (1982). Validity of a statistics attitudes survey: a follow-up study. Educational and Psychological Measurement, 42, 907-912.

Rosenthal, B. (1992). No More Sadistics, no More Sadits, no more vitims. The UMAP Journal, 13(4), 281-289.

Shrigley, R. L., Koballa, Jr., T. R. \& Simpson, R. D. (1988, November). Defining attitude for Science educators, Journal of Research in Science Teaching, 25(8), 659-677.

Silva, C. B., Cazorla, I. M. \& Brito, M. R. F. (1999). Concepções e atitudes em relação à Estatística. Anais da Conferência Internacional: Experiências e perspectivas do ensino de Estatística. Desafios para o século XXI. Florianópolis, versão preliminar, 18-29. 
Silva, C. B., Cazorla, I. M., Vendramini, C. M. M. \& Brito,

M. R. F. (2000). Análise da relação entre os aspectos afetivos e cognitivos com o desempenho em Estatística. Anais do $14^{\circ}$ Simpósio Nacional de probabilidade e Estatística. Caxambu, Minas Gerais, 460.

Spalleta, A. G. (1998). Desenvolvimento das habilidades matemáticas: Um estudo sobre as relações entre o desempenho e a reversibilidade de pensamento durante a solução de problemas (Dissertação de Mestrado). Faculdade de Educação, Universidade Estadual de Campinas, Campinas, São Paulo.

Stagner, R. (1937). Psychology of Personality. New York: McGraw Hill Book Company.

Statistica (1997). Statistica for Windowns, release 5.1. Tulsa, OK: StatSoft Inc.

Vendramini, C. M. M., Silva, C. B., Cazorla, I. M. \& Brito, M. R. F. (2000). Análise da relação entre os aspectos afetivos e cognitivos com o desempenho em Estatística. Anais do $14^{\circ}$ Simpósio Nacional de probabilidade e Estatística. Caxambu, Minas Gerais, 536.
Vendramini, C. M. M. (2000). Implicações das atitudes e das habilidades matemáticas na aprendizagem dos conceitos de Estatística. Tese de doutorado. Faculdade de Educação, Universidade Estadual de Campinas, Campinas, São Paulo.

Waters, I. K., Martelli, T. A., Zakrajset, T. \& Popovic, P. M. (1989). Measuring Attitudes Towards Statistics in a Introductory Course on Statistic. Psychological Reports, 64, 113-114.

Watts, D. G. (1991). Why is Introductory Statistics Difficult to learn? What Can We Do to Make it Easier? The American Statician, 45(4), 290-291.

Wise, S. L. (1985). The development and validation of a scale measuring attitudes toward statistics. Educational and Psychological Measurement, 45, 401-405.

Recebido em: 04/09/2001

Revisado em: 16/10/2001

Aprovado em: 13/12/2001 
ANEXO 1

QUESTIONÁRIO DO ALUNO

NOME:

R.A.:

1. Ano de Ingresso: 19

3. Curso:

5. Período: 1.( ) Manhã

6. Data de Nascimento:

7. Sexo:

1.( ) Masculino

8. Escolaridade do Pai:

1.( ) Nunca estudou

2.( ) $1^{\circ}$ Grau incompleto

3.( ) $1^{\circ} \mathrm{Grau}$ completo

9. Profissão do Pai :

10. Escolaridade da Mãe:

1.( ) Nunca estudou

2.( ) $1^{\circ}$ Grau incompleto

3.( ) $1^{\circ}$ Grau completo

11. Profissão da Mãe :

12. Você já fez ou está fazendo algum curso de Estatística? 1.( )Sim 2.( )Não

Se $\underline{\operatorname{Sim}}$ responda as questões $\underline{13}$. e $\underline{14}$., se Não pule para a questão $\underline{15}$.

13. Onde:1.( ) Colégio 2.( ) Faculdade 3.( ) Empresa 4.( ) Outro local

14. Você já teve reprovações em Estatística?

1.( )Sim. Quantas vezes?

15. Que idéia você tem hoje da Estatística?
2. Série que está cursando:

4. Faculdade:
4.( ) $2^{\circ}$ Grau incompleto
5.( ) $2^{\circ}$ Grau completo
6.( ) Superior
7.( ) Não sei responder

7.( ) Não sei responder

5.( ) $2^{\circ}$ Grau completo

6.( ) Superior

16. Por que motivo estudar Estatística?

17. Cite alguns exemplos de situações do cotidiano em que a Estatística pode ser utilizada.

18. Qual(ais) matéria(s) você já foi reprovado(a), na faculdade ou anteriormente?

1.( ) Nenhuma matéria

2.( ) 1 ou mais matérias (anote todas):

3.( ) Todas as matérias

19. Qual matéria que você menos gosta?

1.( ) Nenhuma matéria

2.( ) Anote apenas uma matéria:

3.( ) Todas as matérias

20. Qual matéria que você mais gosta?

1.( ) Nenhuma matéria

2.( ) Anote apenas uma matéria:

3.( ) Todas as matérias 


\section{ANEXO 2 \\ ESCALA DE ATITUDES EM RELAÇÃo À ESTATíSTICA}

(Aiken e Dreger, 1963); (traduzida e revista por Brito, 1996, 1998)

NOME:

R.A.:

Instruções: Cada uma das frases a seguir expressa o sentimento que cada pessoa apresenta com relação à Estatística. Você deve comparar o seu sentimento pessoal com aquele expresso em cada frase, assinalando um dentre os quatro pontos colocados abaixo de cada uma delas, de modo a indicar com a maior exatidão possível, o sentimento que você experimenta com relação à Estatística. Não deixe nenhuma resposta em branco.

01. Eu fico sempre sob uma terrível tensão na aula de Estatística.

( )Discordo Totalmente ( )Discordo ( )Concordo ( )Concordo Totalmente

02. Eu não gosto de Estatística e me assusta ter que fazer essa matéria.

( )Discordo Totalmente ( )Discordo ( )Concordo ( )Concordo Totalmente

03. Eu acho a Estatística muito interessante e gosto das aulas de Estatística.

( )Discordo Totalmente ( )Discordo ( )Concordo ( )Concordo Totalmente

04. A Estatística é fascinante e divertida.

( )Discordo Totalmente ( )Discordo ( )Concordo ( )Concordo Totalmente

05. A Estatística me faz sentir seguro(a) e é, ao mesmo tempo, estimulante.

( )Discordo Totalmente ( )Discordo ( )Concordo ( )Concordo Totalmente

06. "Dá um branco" na minha cabeça e não consigo pensar claramente quando estudo Estatística.

( )Discordo Totalmente ( )Discordo ( )Concordo ( )Concordo Totalmente

07. Eu tenho sensação de insegurança quando me esforço em Estatística.

( )Discordo Totalmente ( )Discordo ( )Concordo ( )Concordo Totalmente

08. A Estatística me deixa inquieto(a), descontente, irritado(a) e impaciente.

( )Discordo Totalmente ( )Discordo ( )Concordo ( )Concordo Totalmente

09. O sentimento que tenho com relação à Estatística é bom.

( )Discordo Totalmente ( )Discordo ( )Concordo ( )Concordo Totalmente

10. A Estatística me faz sentir como se estivesse perdido(a) em uma selva de números e sem encontrar a saída.

( )Discordo Totalmente ( )Discordo ( )Concordo ( )Concordo Totalmente

11. A Estatística é algo que eu aprecio grandemente.

( )Discordo Totalmente ( )Discordo ( )Concordo ( )Concordo Totalmente 
12. Quando eu ouço a palavra Estatística, eu tenho um sentimento de aversão.

( )Discordo Totalmente ( )Discordo ( )Concordo ( )Concordo Totalmente

13. Eu encaro a Estatística com um sentimento de indecisão, que é resultado do medo de não ser capaz em Estatística.

( )Discordo Totalmente ( )Discordo ( )Concordo ( )Concordo Totalmente

14. Eu gosto realmente da Estatística.

( )Discordo Totalmente ( )Discordo ( )Concordo ( )Concordo Totalmente

15. A Estatística é uma das matérias que eu realmente gosto de estudar na faculdade.

( )Discordo Totalmente ( )Discordo ( )Concordo ( )Concordo Totalmente

16. Pensar sobre a obrigação de resolver um problema estatístico me deixa nervoso(a).

( )Discordo Totalmente ( )Discordo ( )Concordo ( )Concordo Totalmente

17. Eu nunca gostei de Estatística e é a matéria que me dá mais medo.

( )Discordo Totalmente ( )Discordo ( )Concordo ( )Concordo Totalmente

18. Eu fico mais feliz na aula de Estatística que na aula de qualquer outra matéria.

( )Discordo Totalmente ( )Discordo ( )Concordo ( )Concordo Totalmente

19. Eu me sinto tranqüilo(a) em Estatística e gosto muito dessa matéria.

( )Discordo Totalmente ( )Discordo ( )Concordo ( )Concordo Totalmente

20. Eu tenho uma reação definitivamente positiva com relação à Estatística. Eu gosto e aprecio essa matéria.

( )Discordo Totalmente ( )Discordo ( )Concordo ( )Concordo Totalmente

21. Não tenho um bom desempenho em Estatística.

( )Discordo Totalmente ( )Discordo ( )Concordo ( )Concordo Totalmente 